\title{
High strain rate and quasi-static tensile behaviour of Ti-6Al-4V after cyclic damage
}

\author{
J. Galán Lópeza ${ }^{\mathrm{a}}$ P. Verleysen, and J. Degrieck \\ Ghent University
}

\begin{abstract}
It is common that energy absorbing structural elements are subjected to a number of loading cycles before a crash event. Several studies have shown that previous fatigue can significantly influence the tensile properties of some materials, and hence the behaviour of structural elements made of them. However, when the capacity of absorbing energy of engineering materials is determined, fresh material without any fatigue damage is most often used. This study investigates the effect of fatigue damage on the dynamic tensile properties of Ti-6Al-4V in thin-sheet form. Results are completed with tests at quasi-static strain rates and observations of the fracture surfaces, and compared with results obtained from other alloys and steel grades. The experiments show that the dynamic properties of Ti-6Al-4V are not affected by a number of fatigue loading cycles high enough to significantly reduce the energy absorbing capabilities of EDM machined samples.
\end{abstract}

\section{Introduction}

When an engineering material is used in a structure, its energy absorbing capability is assessed using standard testing techniques. However, the conditions in service do not always correspond to the conditions of the tests. Mechanical models take into account a number of parameters which affect the behaviour of the material, such as strain rate and temperature, but ignore any previous cyclic damage. Several studies have shown that fatigue loading deteriorates the mechanical properties of some materials, in some cases even for a low number of cycles. Since a few cyclic loads are almost always unavoidable, in service or during manufacture, its effect on the behaviour of the material, for example in a crash event, has to be taken into account.

The effect of fatigue damage on the mechanical behaviour of materials is in fact a complex phenomenon. The tensile properties of a material can be modified by a cyclic loading history depending on its microstructure and the number and magnitude of the applied alternating loads. Moreover, different effects can be observed on the quasistatic and the dynamic tensile properties.

Several research groups have previously studied the effect of fatigue damage on the tensile properties of materials [1-9], including various steel grades and light alloys. The results of these studies are summarized in table 1 . The table shows which effects were observed on the results of tensile tests at quasi-static and/or high strain rates after a given cyclic load had been applied. While the tensile properties of some materials are strongly affected (steel grades and nickel superalloy $[1,2,7])$, in some others the effect is negligible (some aluminum alloys [4]). Moreover, several experiments have shown that the dynamic behaviour of the material may be affected although no effects are observed in quasi-static tests or these effects are very small (this is the case for some aluminum alloys [8]). It is remarkable that when the behaviour of a material is affected by fatigue damage, the effect can be observed from the earliest stages

a e-mail: Jesus.GalanLopez@UGent.be of fatigue life, although it is more pronounced when the damage level increases.

A common conclusion of all these studies is that the effect of previous fatigue damage on the fracture process appears to be strongly influenced by the microstructure of the material. The rearrangement of dislocations inside the material during cyclic loading can embrittle it and, when the number of loading cycles is high enough, be the origin of fatigue cracks [5]. Since the movement of dislocations is a direct consequence of the particular microstructure of the material, so is its behaviour after cyclic damage. Therefore, the microstructural changes produced by cyclic loading serve as an indicator of how the tensile properties of the material will be affected or not.

This study aims to give some insight into the tensile behaviour of previously damaged Ti-6Al-4V. This multiphase $(\alpha+\beta)$ titanium alloy is a material widely used in industrial applications, due to its combination of mechanical performance and low weight, and also to its capacity to conserve its good properties in extreme conditions, such as high temperatures and corrosive environments.

In addition to fatigue life tests and tensile tests of the as-received material, the properties of damaged specimens will be studied by means of tensile experiments at quasistatic and high strain rates in samples which had been previously subjected to a given number of fatigue loading cycles.

\section{Experimental program}

The experimental procedure consists of machining fatigue test samples from a thin-sheet, subject them to a given number of loading cycles and, finally, extract quasi-static and dynamic tensile specimens from the fatigued samples and perform the corresponding tests, using a Hopkinson bar apparatus for impact experiments and a servohydraulic machine for quasi-static tests. In the process of extracting the tensile specimens from the fatigued samples the edges of the original specimens are removed. Through 
Table 1. Effect of previous fatigue damage on the quasi-static and/or dynamic tensile properties of engineering materials. Horizontal lines separate the results of different research groups.

\begin{tabular}{lcc}
\hline Material & $\begin{array}{c}\text { Effect of fatigue damage on quasi-static } \\
\text { properties }\end{array}$ & $\begin{array}{c}\text { Effect of fatigue damage on dynamic } \\
\text { properties }\end{array}$ \\
\hline 45\# Carbon steel & $\begin{array}{c}\text { Strong degradation [1] } \\
\text { SH4145SQ }\end{array}$ & \\
\hline A6061L, A6061T & & Embrittlement [3] \\
A2219P, A7075P & & Not appreciable [3] \\
\hline HR590 & & Not appreciable [4] \\
\hline 2017-A T3 & & Decrement of fracture energy [5,6] \\
\hline DP600Z, TRIP700Z, & & \\
301LN2B & Small decrement of strength and ductility [8] & $\begin{array}{c}\text { Decrembrittlement [7] } \\
\text { A6061T }\end{array}$ \\
AISI4140T & Significant decrement of strength and ductility [8] & Significant decrement of strength and ductility [8] \\
\hline A6082T & Decrement of ductility [9] & \\
\hline
\end{tabular}

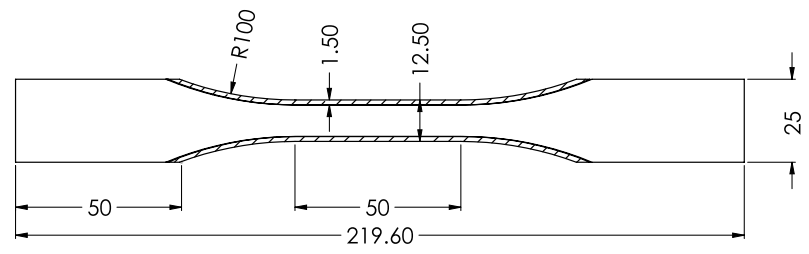

Fig. 1. Specimen geometry for fatigue loading and quasi-static tensile testing. The edges (shaded in the figure) are removed after cyclic loading to avoid the effect of fatigue cracks on tensile tests. See also figure 3 .

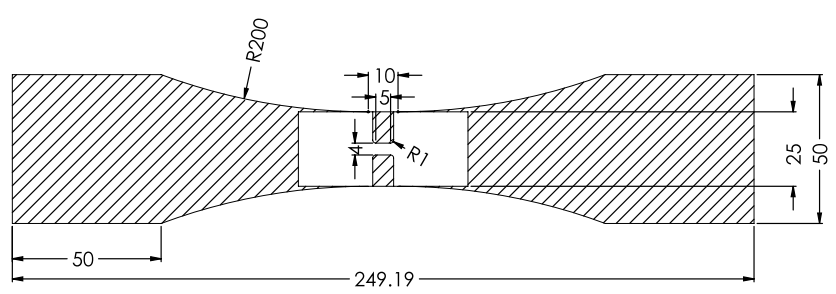

Fig. 2. Specimen geometry for fatigue loading and dynamic tensile testing. The specimen used for the experiments in the Hopkinson apparatus is extracted from the fatigue specimen after cyclic loading. See also figure 3 .

this process, any fatigue cracks originated there are eliminated, so that they do not interfere with the behaviour of the bulk material. In order to confirm that edge cracks indeed are formed and affect the mechanical behaviour of the specimen, a few experiments are performed on damaged samples whose edges had not been removed (due to specimen dimensions constraints, only at quasi-static strain rates).

All the test specimens are machined using spark erosion (also known as electro-discharge machining or EDM) from an annealed sheet with a thickness of $0.6 \mathrm{~mm}$. The rolling direction of the sheet corresponds with the direction in which tensile loads are applied to the samples.

There are normalized rules covering the specimen dimensions required for standard fatigue and quasi-static

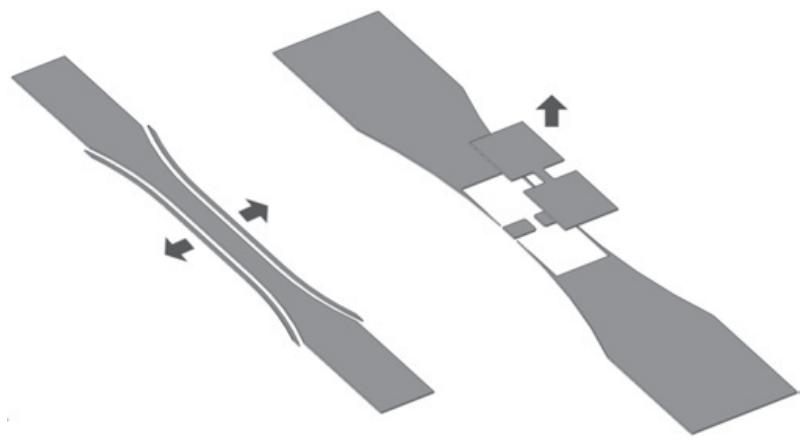

Fig. 3. Removal of edges from fatigued specimens before quasistatic tensile testing (left) and extraction of Hopkinson specimen from larger fatigue samples (right).

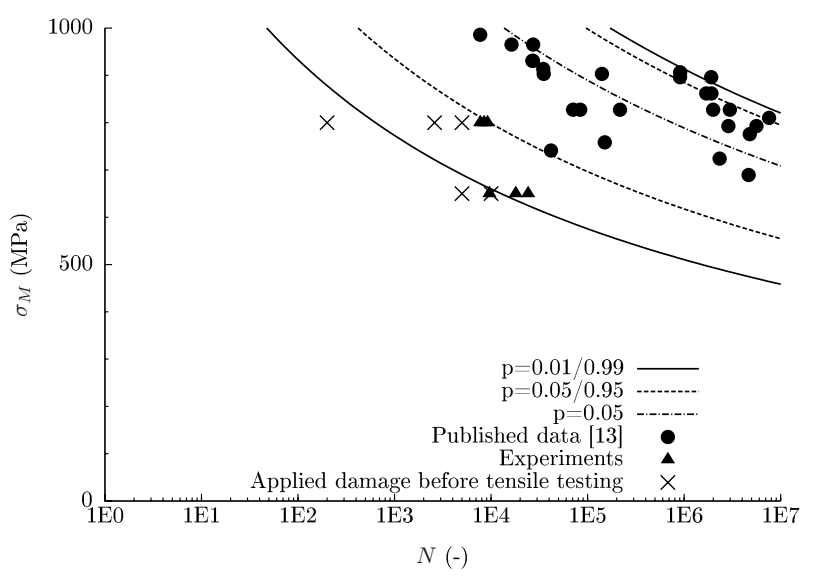

Fig. 4. Fatigue life of the samples used for cyclic loading and quasi-static tests and damage levels applied before tensile testing compared with published fatigue results [13].

tensile testing $[10,11]$. However, it is not possible to design a specimen in agreement with both standards using a sheet of this thickness, so that it can be used for fatigue loading and tensile tests. Therefore, the specimen geometry chosen is a compromise between both sets of rules (figure 1). Dynamic specimens need to have a particular geometry 


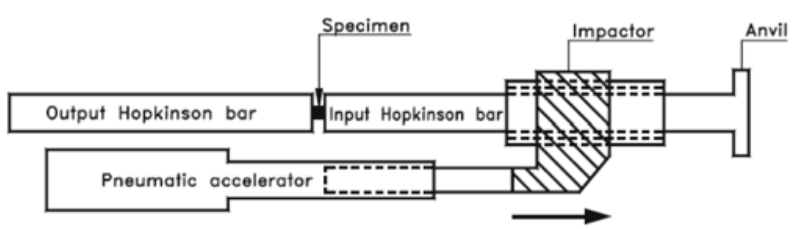

Fig. 5. Schematic view of the split Hopkinson tensile bar setup.
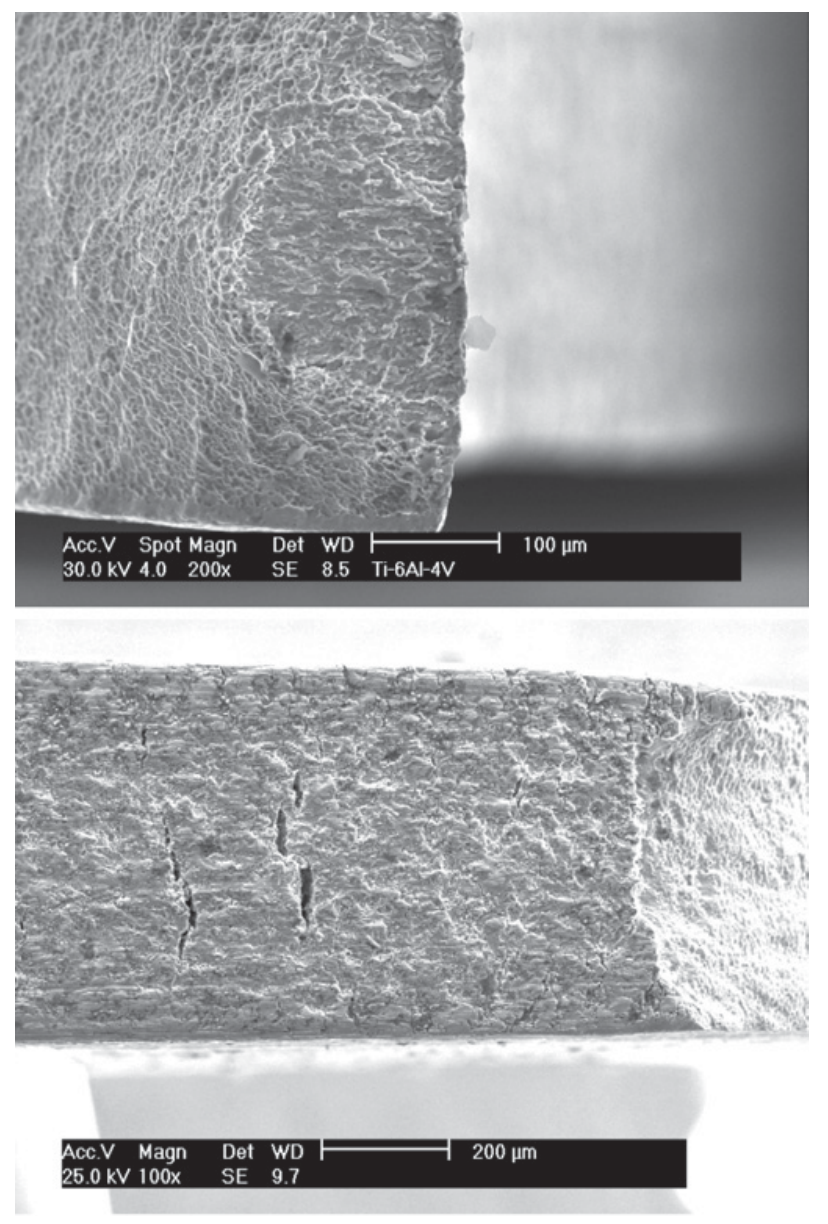

Fig. 6. Top: Edge crack found on the fracture surface of a sample after fatigue failure. Bottom: Similar cracks along the edge of the sample.

[12], so standard fatigue specimens are used for cyclic loading and the smaller Hopkinson specimen is extracted from it after damage has been applied (see figures 2 and 3).

In order to obtain fatigue damaged specimens, cyclic loads are applied with an Instron servo-hydraulic tensile machine. Maximum stress is 800 or $650 \mathrm{MPa}$ and the maximum - minimum stress ratio $(R)$ is 0.1 . These loads are below the yield stress of Ti-6Al-4V (of the order of $1000 \mathrm{MPa}$ ) to avoid plastic deformation, while the stress ratio of 0.1 is chosen to maximize alternating stress without requiring compression loads, which may produce bulking of the specimens.

After determining total fatigue life of the specimens used for quasi-static tests (figure 4) the number of loading cycles to apply is established. When the maximum stress is $800 \mathrm{MPa}$ the selected values are 5000 cycles, which is close to total life, 200 cycles, which is a low value

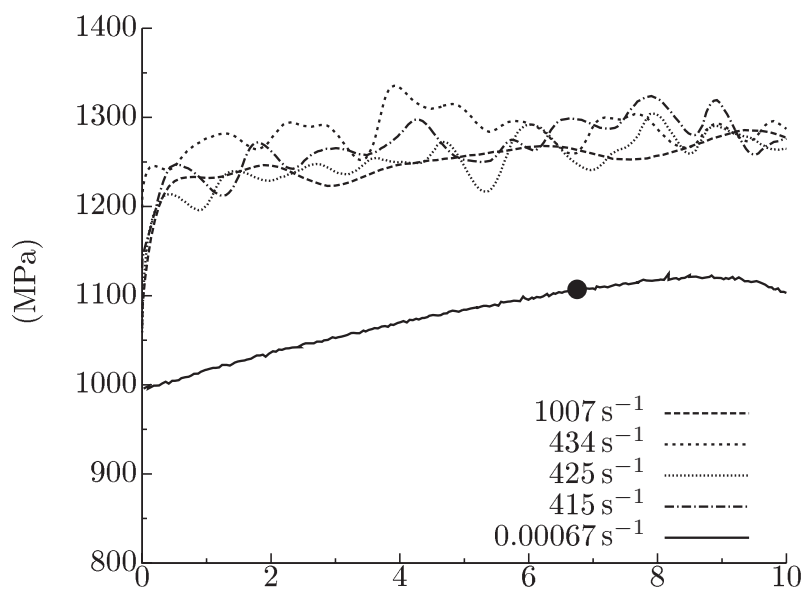

$(\%)$

Fig. 7. True tensile diagram of as-received material at different strain rates. The point indicates the maximum uniform strain.

to observe if there is any early effect, and 2600 , in the middle of these two extreme values. The number of cycles is 5000 and 10000 with the lower stress of $650 \mathrm{MPa}$. Total fatigue life under these loading conditions, measured averaging three experiments, was 8474 for maximum stress of $800 \mathrm{Mpa}$ and 17270 for $650 \mathrm{MPa}$. Specimens for dynamic tests are loaded for 5000 and 10000 cycles with maximum stress of $800 \mathrm{MPa}$.

Quasi-static tensile experiments are performed at strain rate of $0.00067 \mathrm{~s}^{-1}$ (equivalent to a cross-head displacement of $2 \mathrm{~mm} / \mathrm{s}$ ) on the same Instron machine that is used for cyclic loading using damage free specimens and previously fatigued specimens with and without edges.

Dynamic tensile tests at high strain rates are performed on the Split Hopkinson Tensile Bar (SHTB) setup at Ghent University [14] (figure 5). The setup consists of two long bars, an input bar and an output bar (made of aluminum), between which a dog-bone shaped specimen is glued. A tube-like impactor is put around the input bar and is accelerated towards an anvil at the outer end of the bar, generating an incident tensile wave. The incident wave interacts with the specimen and generates a reflected and a transmitted wave. The strain histories corresponding to these three waves, measured by strain gauges on the bars, allow the calculation of the strain rate, strain and stress in the sample using the relations given by Kolsky [15].

\section{Results}

Fatigue life experiments are performed on the specimens of figure 1 in order to determine sensible pre-fatigue levels before tensile tests. Results show that total life is significantly lower than in the tests published in [13] (see figure 4). In contrast to the polished specimens used in [13], samples obtained by EDM have imperfections which function as edge cracks originators [16]. Since the crack initiation stage of fatigue is unnecessary in the presence of these cracks, shown in SEM pictures of figure 6, total life is severely reduced. However, considering that other studies have shown that the effect of fatigue on tensile 
Table 2. Tensile properties at quasi-static strain rate of as-received and previously fatigued Ti-6Al-4V specimens.

\begin{tabular}{lccccccccc}
\hline$\sigma_{M}(\mathrm{MPa})$ & $\sigma_{m}(\mathrm{MPa})$ & $N$ & $N / N_{f}$ & $\mathrm{ER}$ & $\sigma_{u}(\mathrm{MPa})$ & $\varepsilon_{u}(\%)$ & $U\left(\mathrm{~J} / \mathrm{mm}^{3}\right)$ & $q(\%)$ & $\varepsilon_{f}(\%)$ \\
\hline- & - & - & - & No & 1107 & 6.75 & 75 & 28.55 & 33.62 \\
\hline 800 & 80 & 200 & 0.024 & No & 1104 & 6.44 & 71 & 31.18 & 37.37 \\
800 & 80 & 200 & 0.024 & No & 1006 & 6.80 & 76 & 28.90 & 34.11 \\
800 & 80 & 2600 & 0.307 & No & 1087 & 6.33 & 68 & 25.30 & 29.17 \\
800 & 80 & 2600 & 0.307 & No & 1047 & 2.61 & 27 & 26.96 & 31.42 \\
800 & 80 & 5000 & 0.591 & No & 1102 & 6.80 & 80 & 18.58 & 20.56 \\
800 & 80 & 5000 & 0.591 & No & 1024 & 1.52 & 15 & 27.10 & 31.60 \\
800 & 80 & 5000 & 0.591 & No & 987 & 0.00 & 0 & 18.58 & 20.56 \\
\hline 800 & 80 & 2600 & 0.307 & Yes & 1088 & 6.91 & 74 & 29.13 & 34.43 \\
800 & 80 & 2600 & 0.307 & Yes & 1077 & 5.09 & 54 & 28.86 & 34.05 \\
800 & 80 & 2600 & 0.307 & Yes & 1105 & 6.73 & 72 & 29.04 & 34.30 \\
800 & 80 & 5000 & 0.591 & Yes & 1099 & 7.21 & 80 & 28.77 & 33.92 \\
800 & 80 & 5000 & 0.591 & Yes & 1101 & 7.05 & 82 & 29.54 & 35.01 \\
800 & 80 & 5000 & 0.591 & Yes & 1120 & 7.26 & 78 & 28.58 & 33.66 \\
650 & 65 & 5000 & 0.290 & Yes & 1109 & 6.38 & 70 & 29.36 & 34.75 \\
650 & 65 & 5000 & 0.290 & Yes & 1106 & 6.52 & 72 & 30.40 & 36.25 \\
650 & 65 & 5000 & 0.290 & Yes & 1094 & 6.67 & 72 & 26.26 & 30.46 \\
650 & 65 & 10000 & 0.579 & Yes & 1102 & 6.48 & 68 & 32.22 & 38.90 \\
650 & 65 & 10000 & 0.579 & Yes & 1108 & 7.00 & 76 & 29.77 & 35.34 \\
\hline
\end{tabular}
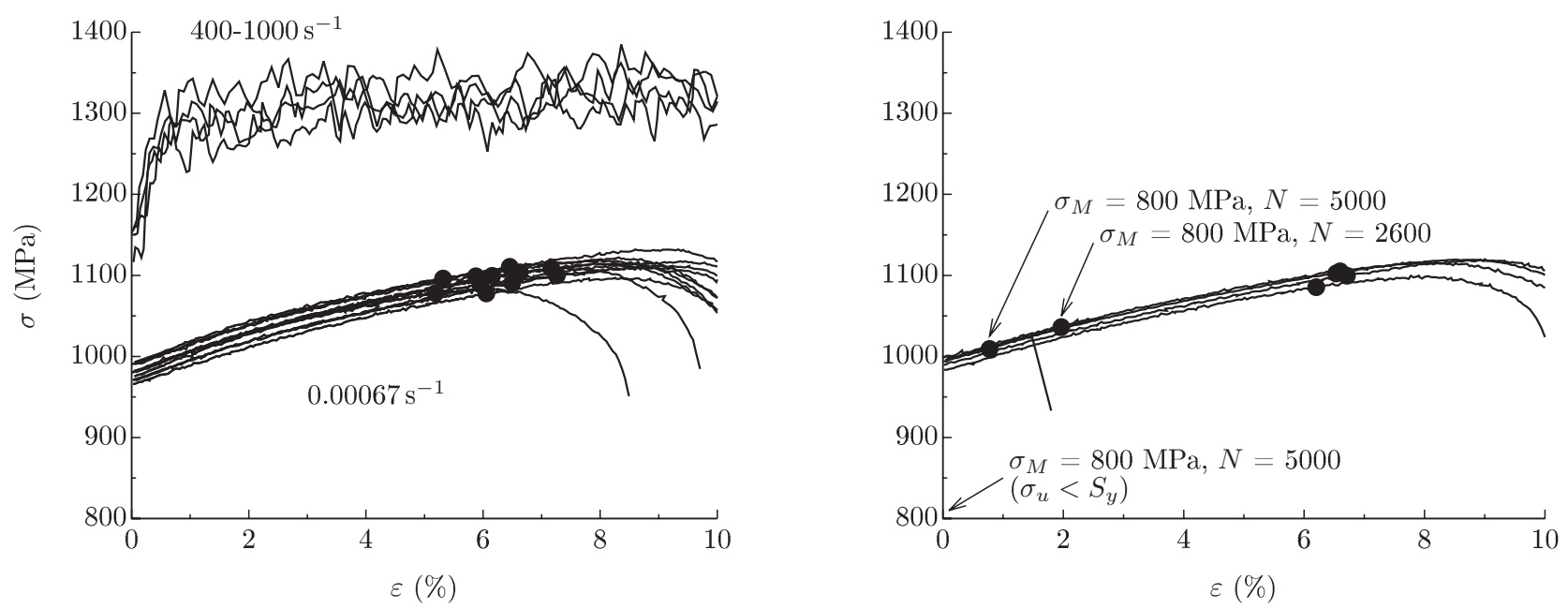

Fig. 8. True tensile diagram of previously fatigued material at different strain rates with edge removal (left) and without it (right).

properties can be observed since the earliest stages of total life, the performance of EDM specimens is acceptable for the purposes of this study.

Figure 7 shows true tensile diagrams of as-received material at quasi-static and impact strain rates (oscillations in the high strain rate diagrams are experimental scatter unavoidable due to the testing method). Tensile properties at quasi-static strain rate are also shown in table 2 . In this table, fracture strain does not correspond to the last measured strain, since experimental data is not reliable when there is no uniform elongation. Instead, it is calculated from the area reduction measured in the fracture surface of the broken samples.

It is observed that Ti-6Al-4V is a material sensitive to strain rate. Yield and ultimate stress increase with higher deformation speeds, while strain hardening is reduced.

\subsection{Effect of pre-fatigue on quasi-static tensile properties}

The tensile curves of previously fatigued specimens (left of figure 8 and table 2) show no significant differences with the diagrams of as-received material (figure 7). This result indicates that the quasi-static tensile properties of Ti-6Al$4 \mathrm{~V}$ are not affected by the fatigue loads applied.

When the edges of the samples are not removed after cyclic loading (figure 8, right), some of the samples suddenly fail at lower levels of strain. Table 2 and figure 10 show that both maximum uniform strain and fracture strain are significantly reduced. It is noticed that this reduction do not have a direct correlation with the number or magnitude of the fatigue load applied. Such a random result is related to the formation of cracks, random in nature. Indeed, a 

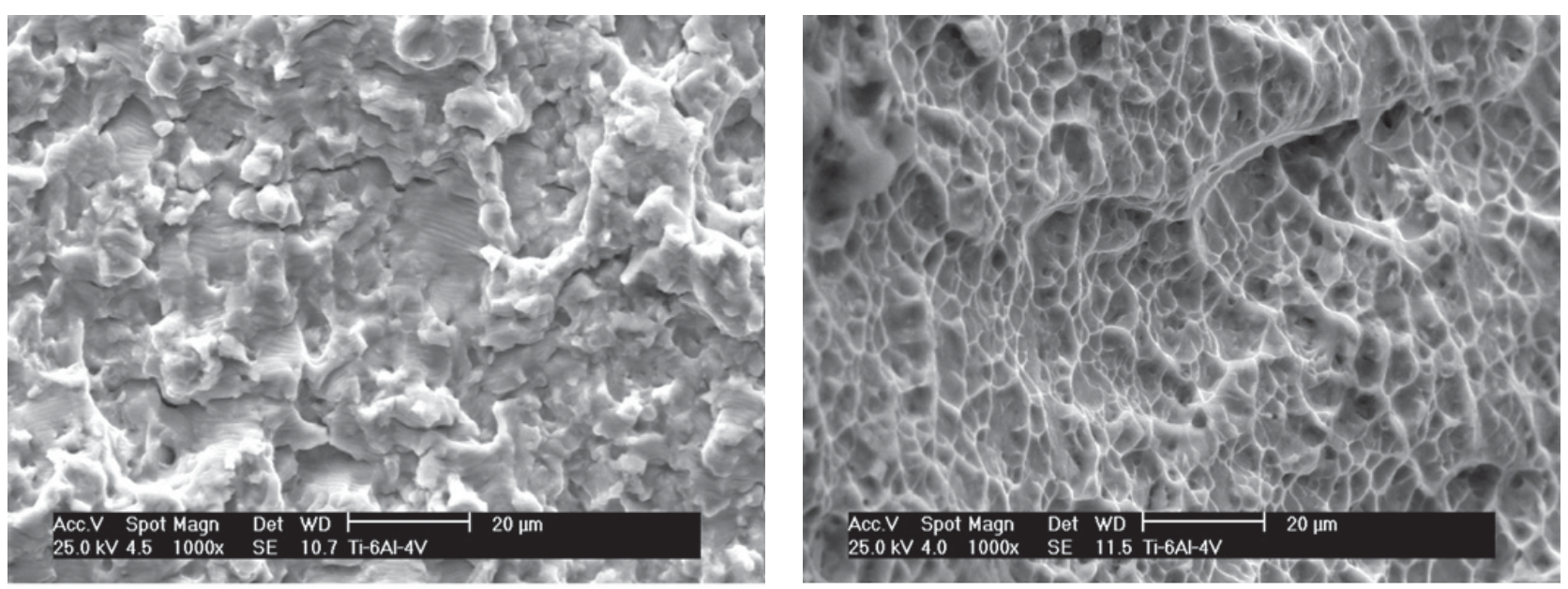

Fig. 9. Fatigue striations are found near the edge of the samples after fatigue failure (left), but the fracture is completely ductile around the middle of the specimen (right).
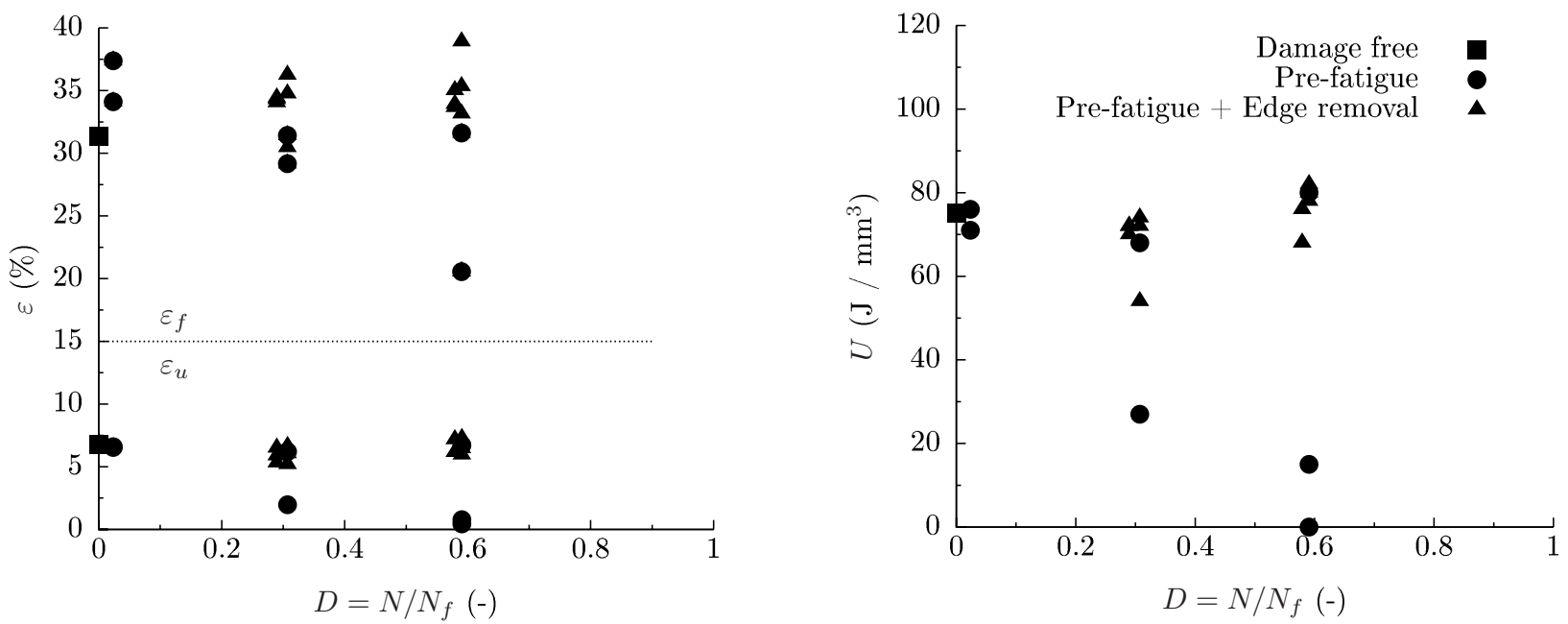

Fig. 10. Left: Uniform and fracture strain of damaged specimens. Right: Deformation energy absorbed during uniform elongation.

close observation of the failed fatigue specimens (figure 6) shows that edge cracks are being formed in the specimens during cyclic loading.

Although cracks are found on the edges of fatigued samples, the central regions of the specimens do not show any specific fatigue features. Figure 9 shows the difference in the fracture surface of a sample which failed at the end of its life in a fatigue experiment. Although the fracture surface is characteristic of fatigue failures near the edges of the sample, the central region of the specimen presents a completely ductile fracture. This observation confirms results from tensile tests with or without edges: fatigue damage is being accumulated near the edges of the specimen, but there is no significant effect on the bulk material.

Since the premature failure of samples is determined by the presence or not of damaged edges, it can be concluded that sudden fracture is not due to a change in the mechanical properties of the material, as it has been observed, for example, in tests of the GH4145/SQ alloy [2] or AISI 4140 steel [8]. Instead, Ti-6Al-4V can be considered part of the group of unaffected materials, similarly to most of the aluminum alloys previously studied [8].

\subsection{Effect of pre-fatigue on dynamic tensile properties}

Results of experiments at high strain rate are more difficult to compare due to the oscillations present on the signal. However, it is clearly observed that there is no significant effect of previous damage on the experimental results. Since the samples tested at high strain rate do not have edge cracks present due to the procedure followed to extract the specimen for the high strain rate test from the fatigued sample, the similarity of the curves of damagefree and fatigued specimens indicates that the applied damage is not having any effect on the bulk material.

This result sets Ti-6Al-4V apart from the aluminum alloys whose dynamic properties are degraded after cyclic loading [3,8]. However, similar results have been found for some particular aluminum and magnesium alloys (A6061PT6 and 5454-O) [6,8].

\section{Conclusions}

The interaction between cyclic loading and tensile properties is a complex phenomenon, dependent of numerous 
factors and with different results depending on the studied material.

Tensile experiments performed on Ti-6Al-4V have not shown any significant difference in the properties of prefatigued specimens and damage-free material. Both quasistatic and dynamic tensile properties appear to be insensitive to the cyclic loads applied in this study. However, if fatigue cracks are allowed to grow on the surface, as it happens when imperfections introduced in the cutting method are present, the fracture strain and the energy absorbed during deformation are substantially, but randomly, reduced.

\section{References}

1. Ye D.Y. and Wang Z.L., Mater. Sci. Eng. A - Struct. Mater. Prop. Microstruct. Process. A297, (2001) 5461

2. Ye D.Y. and Wang Z.L., Int. J. Fatigue 27, (2005) 1102-1114

3. Itabashi M. and Fukuda H., Impact Engineering and Application (2001) 433-438

4. Kobayashi H., Daimaruya M., Tsuda H., Horikawa K. and Yamada Y., J. Mod. Phys. B 22, (2008) 1141-1146

5. Froustey C. and Lataillade J. L., Mater. Sci. Eng. A Struct. Mater. Prop. Microstruct. Process. 500, (2009) 155-163
6. Auzanneau T., Froustey C. and Lataillade J. L., Fatigue Fract. Eng. Mater. Struct. 24, (2001) 23-31

7. Verleysen P., Vanduynslager P., Van Slycken J., Vermeulen M. and Degrieck J., J. Phys. IV 134, (2006) 1307-1312

8. Sánchez-Santana U. Rubio-González C., Mesmacque G., Amrouche A. and Decoopman X., Int. J. Fatigue 30, (2008) 1708-1719

9. Cadenas-Herrera P., Amrouche A., Mesmacque G., Jozwiak K. and Puchi-Cabrera E. S., Fatigue Fract. Eng. Mater. Struct. 33, (2010) 54-65

10. ASTM International, ASTM Standard E466-96: Standard Practice for Conducting Force Controlled Constant Amplitude Axial Fatigue Tests of Metallic Materials (ASTM, USA 1996)

11. ASTM International, ASTM Standard E8M-04: Standard Test Methods for Tension Testing of Metallic Materials [Metric] (ASTM, USA 2004)

12. Verleysen P., Degrieck J., Verstraete T. and Van Slycken J., Exp. Mech. 48, (2008) 587-598

13. Rhode T.M. and Ertel P. W., Metallic Materials Properties Development and Standardization. DOT/FAA/AR-MIL-HDBK-5 (National Technical Information Service, USA 1988)

14. Verleysen P. and Degrieck J., J. Phys. IV 110, (2003) 501-506

15. Kolsky H., Proc. Phys. Soc. B 62, (1949) 676-700

16. Novovic D., Dewes R. C., Aspinwall D. K., Voice W. and Bowen P., Int. J. Mach. Tool. Manu. 44, (2004) $125-134$ 